\title{
Controllable pitch propeller system using the shaft line energy Teodor POPA ${ }^{1, a *}$ and Ovidiu Sorin CUPSA ${ }^{2, b}$ \\ ${ }^{1}$ Ovidius University, Faculty of Mechanical, Industrial and Maritime Engineering, Blvd. Mamaia, No. 124, 900527, Constanta, Romania \\ ${ }^{2}$ Constanta Maritime University, Faculty of Navigation and Naval Transport, Mircea cel Batran Str., No. 104, 900663, Constanta, Romania) \\ ateodorpopa@ceronav.ro; bovidiucupsa@ceronav.ro
}

Keywords: controllable pitch, propeller, pitch control mechanism, shaft, propulsion.

\begin{abstract}
The newest technologies in the maritime field have gained a special dynamics over the last years. In particular it has been sought the improvement of the systems, procedures and rules related to environmental protection, but at the same time, lowering the fuel consumption has been targeted through various residual energy recovery methods, with an eye on lowering gas emissions in the atmosphere, in general, and $\mathrm{CO}_{2}$ in particular.

This paper is aimed at just this target and it proposes a new controllable pitch propeller changing mechanism to be analysed, that would lead to lowering of energy consumption and by default to a reduction in the fuel consumption used by the diesel-generator.
\end{abstract}

\section{Introduction}

Any propulsion unit represents a system that transforms the mechanical energy of a certain movement into mechanical energy of a different movement. Specifically, the ship propeller transforms the power installed on board ship into a thrusting force necessary to overcome the ship's resistance to forward movement, ensuring it's shifting with a certain imposed speed [3].

Specific to the ship propeller is the fact that it works in the ship's wake, therefore in an unevenly, turbulent fluid current, and the cavitation phenomenon imposes a certain specific geometry propeller, otherwise it would lead in yield loss $[3,5]$.

It is known that the ship propeller is characterized by propulsion yield, pitch distribution, blade shape, and blades' positions related to the propeller's axis and the cavitation phenomenon.

\section{Materials and experimental procedure}

\section{Calculation of the angular rotation of the blade}

Calculation of controllable pitch propeller mechanism is made in several stages provided that some technical details are known: the mechanism from the inside of propeller hub, hydraulic cylinder strengths, blocking differential mechanism strengths from the hydraulic pump, the clutch between shaft and differential mechanism and the propeller shaft.

To avoid the overloading of the engine we shall calculate the torque coefficient $\mathrm{k}_{\mathrm{Q}}$, under whose value we will not go down, the advance number of the propeller $\mathrm{J}$ and coefficient $\mathrm{k}_{\mathrm{SH}}=\mathrm{f}(\mathrm{J})$, for angle $\theta$.

Table 1

\begin{tabular}{|l|l|l|}
\hline$\theta\left[{ }^{\circ}\right]$ & $\mathrm{J}$ & $\mathrm{k}_{\mathrm{SH}}$ \\
\hline-10 & 0,08 & $-0,009$ \\
\hline 0 & 0,5 & 0,005 \\
\hline 10 & 0,92 & 0,01 \\
\hline 15 & 114 & 0,007 \\
\hline
\end{tabular}


With these values we draw the curve $\mathrm{kSH}$ obtained for the torque coefficient $\mathrm{kQ}=0,017$, pitch/diameter ratio $\mathrm{H} / \mathrm{D}=0.8$ and the projected area ratio $\mathrm{A} / \mathrm{AD}=0.65$, resulting a moment of maximum blade rotation, $\mathrm{kShmax}=0,0101$, which will be taken into calculations to avoid engine overloading [1,2].

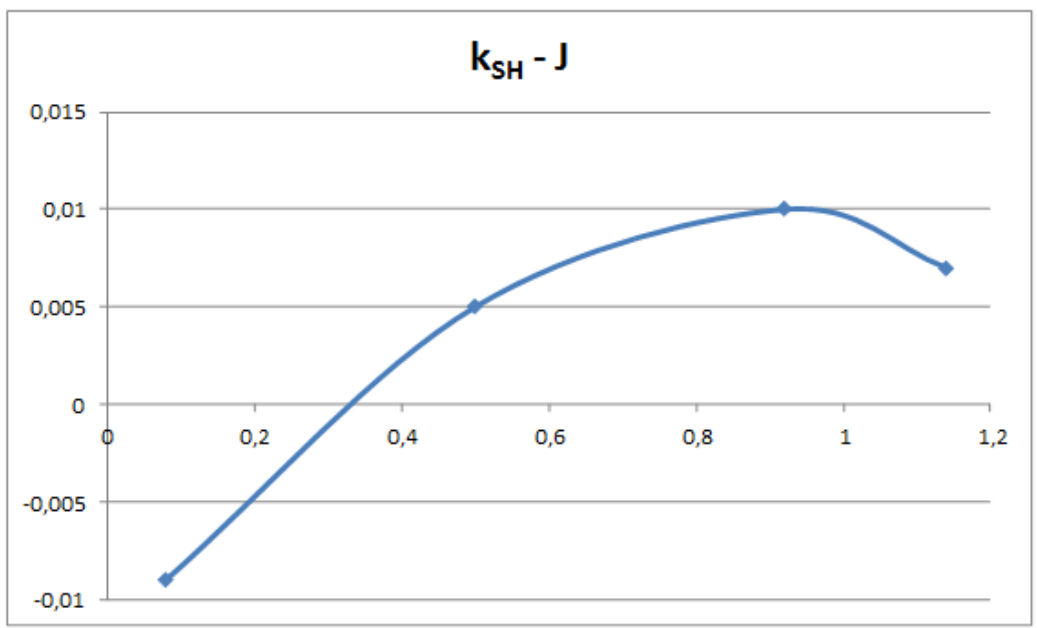

Fig. 1 Diagram of advanced number and coefficient of rotation moment

The time of blade rotation, tested in the hydrodynamic tunnel has three components:

$Q_{s}=Q_{s c}+Q_{v h}+Q_{s f}$

where:

Qsc - moment caused by centrifugal forces; Qvh - moment due to hydrodynamic strengths; Qsf - moment due to friction forces

The measurements are made for three blade propellers, for four blades will result:

$Q_{s h}=\frac{Q_{s h}}{3} \cdot 4=\frac{4125,44}{3} \cdot 4=5500,58 \mathrm{kgf} \cdot \mathrm{m}$

\section{Calculation of force transmission}

For the calculation of force transmission it will be necessary to consider the kinematics scheme which will determine the Fp force.

For calculation of control rod strength, driven by hydraulic cylinder we shall considered that a unitary force acts upon the sliding roller. In this case, the rotation moment of blade propeller corresponding to the unitary force will be:

$\mathrm{M}=\mathrm{F} \cdot \mathrm{b}$

Where:

$\mathrm{F}$ - is the resultant of the forces acting on the sliding roller

After processing the data, it will result that the maximum force is [5]:

$\mathrm{F}_{\text {pmax }}=13172 \mathrm{kgf}$

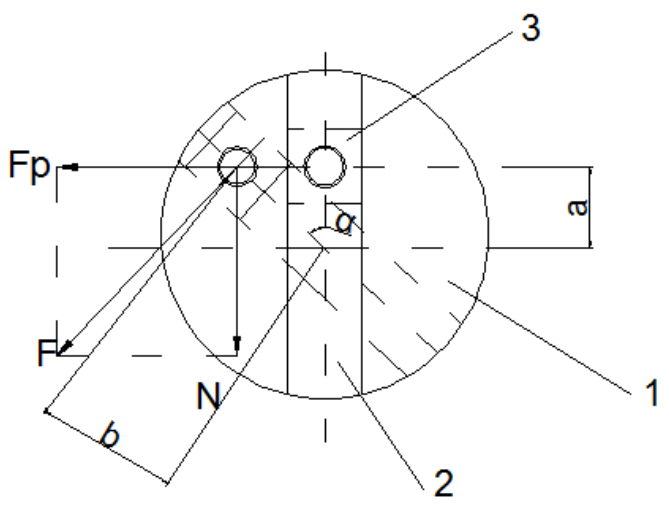

Fig. 2 The kinematic scheme of pitch propeller mechanism 


\section{Calculation of hydraulic cylinder}

Cylinder volume will be:

$V_{c i l}=c \cdot \frac{\pi \cdot d^{2}}{4}=\frac{0,235 \cdot \pi \cdot(0,13)^{2}}{4}=3,119 \cdot 10^{-3} \mathrm{~m}^{3}$

\section{Calculation of clutch}

The torsion moment to calculate the clutch is given by the relation:

$M_{\text {max }}=\frac{\beta \cdot M_{r}}{k_{m} \cdot k_{v}}$

where

$\beta$ - substitution coefficient

$\beta=1,3$

$\mathrm{k}_{\mathrm{m}}$ - coefficient which considers the number of couplings per hour

$\mathrm{k}_{\mathrm{m}}=0,9$

$\mathrm{k}_{\mathrm{v}}-$ coefficient which considers the tangential speed on the medium-diameter circle

$\mathrm{k}_{\mathrm{v}}=0,8$

$\mathrm{M}_{\mathrm{r}}-$ the moment of resistant forces calculated at differential mechanism

$\mathrm{M}_{\mathrm{r}}=390,189 \mathrm{~N} \cdot \mathrm{m}$

$M_{\text {max }}=\frac{1,3 \cdot 390,189}{0,9 \cdot 0,8}=705 \mathrm{~N} \cdot \mathrm{m}$

Axial force is:

$F_{a}=\frac{3 \cdot M_{\max }}{\mu} \quad \frac{D_{2}^{2}-D_{1}^{2}}{D_{2}^{3}-D_{1}^{3}}$

where:

$\mu-$ friction coefficient

$\mu=0,3$

$\mathrm{D}_{1}, \mathrm{D}_{2}$ - outside and inside diameters of cluch abrasive surface

$$
\begin{aligned}
& \mathrm{D}_{1}=840 \mathrm{~mm} \\
& \mathrm{D}_{2}=920 \mathrm{~mm}
\end{aligned}
$$

Verification of the clutch is made by comparing real contact pressure with admissible contact pressure ( $\mathrm{pa}=0,20 \mathrm{~N} / \mathrm{mm} 2)$. Contact pressure will be:

$$
\begin{aligned}
& p=\frac{F_{a}}{\frac{\pi}{4} \cdot\left(D_{2}^{2}-D_{1}^{2}\right)} \leq p_{a} \\
& p=\frac{5337,23}{\frac{\pi}{4} \cdot\left(920^{2}-840^{2}\right)}=0,048 \mathrm{~N} / \mathrm{mm}^{2} \leq p_{a}
\end{aligned}
$$

\section{Calculation of pneumatic motor}

Membrane active diameter is:

$D=k \cdot \sqrt{\frac{F}{p}}$

$\mathrm{k}-$ coefficient

$\mathrm{k}=13-$ for cylindrical membranes

$\mathrm{F}$ - rod force [ daN ]

$\mathrm{p}$-air pressure $\left[\mathrm{daN} / \mathrm{cm}^{2}\right]$ 
$D=13 \cdot \sqrt{\frac{533,7}{6}}=141,4 \mathrm{~mm}$

Maximum stroke of membrane is $\mathrm{C}_{\max }=0,2 \mathrm{D}=0,2 \cdot 150=30 \mathrm{~mm}$.

And rod force is $561,8 \mathrm{daN}$.

\section{Calculation of differential mechanism}

The role of the differential mechanism is to change the direction of pumps rotation and implicitly the direction of fluid flow through the pump to the hydraulic cylinder.

Kinematic scheme of differential mechanism is:

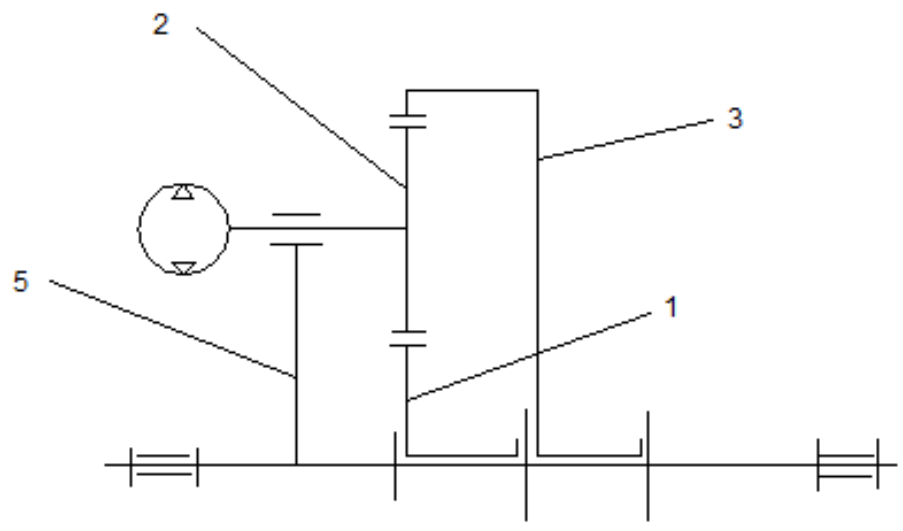

Fig. 4.13 Differential mechanism

1-Toothed wheel; 2 - pinion 3 - Toothed wheel

Mechanism mobility is:

$$
\mathrm{M}=2
$$

The number of teeth for pinion 2 is chosen at the subcut limit $\mathrm{z}_{2}=17$.

If toothed wheel 3 is blocked, then:

$\mathrm{z}_{1}=209$

$\mathrm{Z}_{2}=17$

$\mathrm{Z}_{3}=243$ și $\omega_{3}=0$

According [4], the module is:

$$
\begin{aligned}
& m=\sqrt[3]{\frac{2 \cdot M_{t 1} \cdot \cos ^{2} \alpha \cdot c_{F} \cdot K_{C}^{2} \cdot K_{M}^{2}}{z_{2} \cdot \cos ^{2} \alpha_{0} \cdot \psi_{m} \cdot \sigma_{H a}^{2}} \cdot \frac{i_{21}+1}{i_{21}}} \\
& m=\sqrt[3]{\frac{2 \cdot 39019 \cdot 1,716 \cdot \cos 21^{\circ}}{42 \cdot 17 \cdot \cos 20^{\circ} \cdot 6} \cdot 2,01}=3,96
\end{aligned}
$$

The module is 4

Torsion moment

$$
M_{t 1}=\frac{P[\mathrm{~kW}]}{\omega_{1}}=\frac{1,36 \cdot 75 \cdot 9,81 \cdot 7,5}{\frac{1154}{60}}=390,189 \mathrm{~N} \cdot \mathrm{m}
$$

$\mathrm{C}_{\mathrm{F}}-$ Coefficient in case of pressure contact stress

$\mathrm{C}_{\mathrm{F}}=\mathrm{k}_{\mathrm{s}} \cdot \mathrm{k}_{\mathrm{v}} \cdot \mathrm{k}_{\mathrm{Hb}}$

$\mathrm{k}_{\mathrm{s}}$ - outside pressure factor $\left(\mathrm{k}_{\mathrm{S}}=1,5\right)$

$\mathrm{k}_{\mathrm{v}}$ - internal dynamic factor $\left(\mathrm{k}_{\mathrm{v}}=1,3\right)$

$\mathrm{k}_{\mathrm{Hb}}-$ distribution factor $\left(\mathrm{k}_{\mathrm{Hb}}=1,1\right)$ 


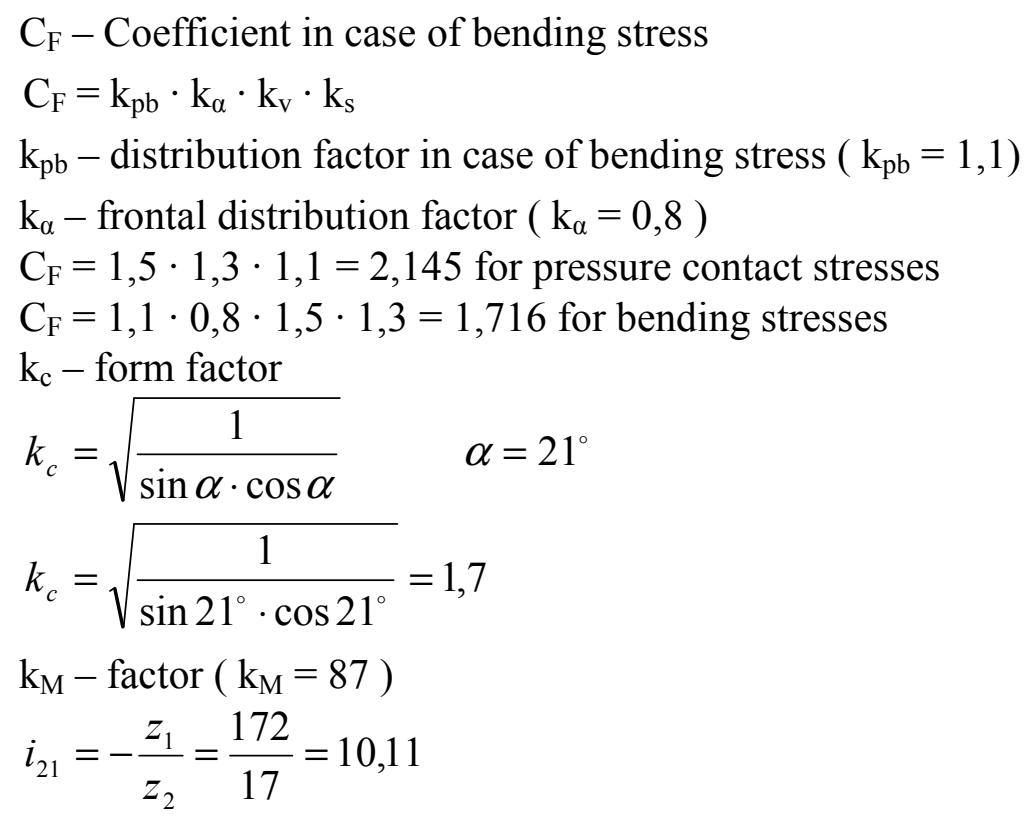

\section{Results and discussions}

The paper presents the calculus elements for the blade's torque values, for the control rod as well as for the duration of the blade rotation.

As well calculus elements for the main components of the proposed system for changing the pitch for the controllable blade propeller are presented. Thus are shown the values resulting from calculating the hydraulic cylinder, clutch, command pneumatic engine, differential mechanism, shaft line, intermediate shaft, propeller shaft, the joints between shafts and thrust bearing.

\section{Conclusions}

The adjustable pitch propeller possesses a large number of advantages, beyond its high complexity in comparison with the constant pitch propeller alternative. Clearly manoeuvrability is one of the advantages, the smooth control of the propulsion can be realised without accelerating or decelerating the engine. Beyond that, the high control of the propulsion is important in some important situations while manoeuvring a vessel: for example mooring manoeuvres, binding to a quay, anchoring, passing through channels, straits, locks or binding to another vessel.

The propeller can function with minimum or without any energy losses. The forth or backwards direction can be changed within minutes or even seconds, depending on how much cargo is on board or on the propeller loading condition. This fact not only does it help absorb all the power generated by the engine but it also helps preventing fuel loss.

\section{References}

[1] T. Popa, A. Pintilie - Marine shipboard installation, Ed Muntenia, Constanta, 2004.

[2] J. Popovici, V. Ceanga - Propeller calculus. Naval propeller, Ed Academiei Romane, Bucuresti, 1990.

[3] A. Molland - The Maritime Engineering Reference Book, 1st Edition - A Guide to Ship Design, Construction and Operation, 2008.

[4] A. Chisiu, D.Matiesan, T. Madarasan, D. Pop - Machine parts - Ed. Didactica si Pedagogica, Bucuresti, 1981.

[5] I. Ionita, J. Apostolache - Naval systems, Editura Tehnica, Bucuresti, 1986. 